\title{
Beneficial effects of maltobionic acid on bone density in healthy Japanese adult women: A randomized double-blind placebo- controlled study
}

\author{
Daiki Suehiro $^{1}$, Ken Fukami ${ }^{1}$, Tsuyoshi Takara ${ }^{2}$ \\ ${ }^{1}$ San-ei Sucrochemical Co., Ltd., 24-5, Kitahama-mac hi, Chita-city Aichi, 478-8503, Japan; \\ ${ }^{2}$ Medical Corporation Seishinkai, Takara Clinic, 9F Taisei Building, 2-3-2, Higashi-Gotanda, \\ Shinagawa-ku, Tokyo 141-0022, Japan
}

Corresponding author: Daiki Suehiro, Research and Development department, San-ei Sucrochemical Co., Ltd. 24-5, Kitahama-machi, Chita-city Aichi, 478-8503, Japan

Submission Date: June $18^{\text {th }}$, 2019. Acceptance Date: September 27 ${ }^{\text {th }}$, 2019. Publication Date: September $30^{\text {th }}, 2019$.

Citation: Daiki Suehiro, Ken Fukami, Tsuyoshi Takara. Beneficial effects of maltobionic acid on bone density in healthy Japanese adult women: A randomized double-blind placebo-controlled study. Functional Foods in Health and Disease 2019; 9(9): 606-627. DOI: https://doi.org/10.31989/ffhd.v9i9.629

\begin{abstract}
Background: Osteoporosis is characterized by reduced bone mineral density (BMD) and increased fracture risk, with a higher incidence in post-menopausal women. This study aimed to evaluate the safety and efficacy of long-term ingestion of maltobionic acid on BMD in healthy Japanese women.
\end{abstract}

Methods: A randomized, double-blind, placebo-controlled, parallel-group study was conducted from February to December 2018. Thirty-eight healthy Japanese women aged 50-69 years who were at least 1 year past the onset of natural menopause were allocated to two groups (19 in each group) using a computerized random-number generator: one in which participants ingested $7 \mathrm{~g}$ of corn syrup containing maltobionic acid and another in which participants ingested $7 \mathrm{~g}$ of placebo (maltose syrup) per day for 24 weeks. BMD and bone metabolism parameters were measured dualenergy X-ray absorptiometry (DEXA) method and a peripheral blood test, respectively, whereas safety was evaluated via a physical examination, peripheral blood test, urinalysis, assessment of subjective symptoms, and a medical questionnaire.

Results: Of the 38 subjects, one subject discontinued the study halfway and 14 were excluded before the efficacy analysis because of conflicts with control criteria. Thus, the final study 
population was 23 subjects (10 in the Test food group and 13 in the Placebo group). There were no adverse events related to consumption of the test food. Consumption of corn syrup solids containing maltobionic acid was maintained during the intervention period, and BMD, bone mineral content, and young adult mean values were found to be improved $(P<0.05)$. No safety concerns were observed during the intervention period.

Conclusion: These results indicate that the consumption of maltobionic acid may contribute to the prevention of osteoporosis.

Trial registration: UMIN-CTR ID: UMIN000031489; Foundation: San-ei Sucrochemical Co., Ltd.

Keywords: Maltobionic acid; bone mineral density; safety; long-term intake; osteoporosis; menopause

\section{BACKGROUND}

Osteoporosis is defined by WHO as "A disease characterized by low bone mass and microarchitectural deterioration of bone tissue, leading to enhanced bone fragility and a consequent increase in fracture risk" [1]. With the aging society in Japan, the number of patients with osteoporosis is increasing annually, and the prevalence of osteoporosis was estimated to be approximately 13 million in 2015 [2,3]. Osteoporotic fractures are associated with poorer quality of life [4] and increased risk of mortality [5]. Bone mineral density (BMD) is an important predictor of osteoporotic fractures [6], and sufficiency of everyday intake of nutrients such as calcium, vitamin $\mathrm{D}$, and vitamin $\mathrm{K}$ are known to have a major impact on BMD. Calcium in particular is a major mineral component of bone. Calcium intake has been found to be significantly related to bone mineral content (BMC) and BMD [7-9]; appropriate intake of this mineral is, therefore, important for the prevention of osteoporosis. In addition to the significance of the amount of calcium consumed, its efficient absorption by the intestines is important for improving nutritional status. The absorbability of calcium is influenced by various factors including the form in which calcium is ingested and its associated properties, such as its solubility, and the food consumed with it (e.g., oxalic acid and phosphorus) [10, 11].

Maltobionic acid (4-O- $\alpha$-D-Glucopyranosyl-D-gluconic acid: CAS No. 534-42-9), in which glucose is $\alpha$-1,4-bonded to gluconic acid, is an indigestible disaccharide present in honey. Maltobionic acid also forms a stable salt with inorganic cations that maintains high water solubility even when ion-bound with calcium [12]. We have previously reported that maltobionic acid enhances calcium and magnesium absorption and increases the amount of calcium in rat femurs by maintaining the solubilized state of minerals throughout the intestinal tract $[12,13]$. Furthermore, in a 24-week intervention study in postmenopausal women, the intake of calcium salts composed mainly of maltobionic acid was shown to maintain and increase BMD, with no safety concerns related to their long-term consumption [14]. However, there are no reports on BMD improvement resulting from intake of the mineral-free state of maltobionic acid or information on the safety of its long-term intake. In the present study, we investigated the safety and efficacy of the long-term ingestion of maltobionic acid on the BMD of healthy Japanese women. 


\section{METHODS}

\section{Study design and participants}

This was a randomized double-blind placebo-controlled study. The participants were recruited by ORTHOMEDICO Inc. (Tokyo, Japan), which runs a clinical trial recruitment site known as Go106 (https: //www.go106.jp). Individuals interested in participating were given a full explanation of the study. Those who provided written consent underwent preliminary selection process. Individuals who met the following criteria were included: Japanese women aged 50-69 years who were at least 1 year past the onset of natural menopause. Those who met any of the following exclusion criteria were excluded: (a) history of treatment for malignant tumor, cardiac failure, or myocardial infarction; (b) presence of other diseases (arrhythmia, liver dysfunction, kidney dysfunction, cerebrovascular disease, rheumatism, diabetes, dyslipidemia, hypertension, or other chronic disease); (c) regular use of pharmaceutical drugs (including kampo) or supplements; (d) regular ingestion of foods for specialized health use or with functional claims; (e) ingestion at least once per week of calcium, vitamin D, vitamin K, magnesium, isoflavones (including daidzein, genistein, equol) and all other supplements, foods for specialized health use, foods with functional claims, and foods with nutritional function claims that may affect bone metabolism; (f) allergies (pharmaceuticals and foods related to the test foods in this study); (g) participation in another clinical study within 3 years of providing written consent to participate in the present study; or (h) any other reason the principal investigator found to disqualify the individual from participating in this study.

This study's protocol received approval from the Institutional Review Board of Takara Clinic (Tokyo, Japan) on February 20, 2018 (no. 1802-1712-ST01-03-TC). The study was conducted with full consideration of medical ethical principles and in accordance with the Declaration of Helsinki (2013) and the Ethical Guidelines for Medical and Health Research Involving Human Subjects. Testing was mainly conducted by the Takara Clinic. This study was registered with the University Hospital Medical Information Network (UMIN000031489). Subjects were recruited from February 27 to April 28, 2018, and the study was conducted from May 28 to December 1, 2018.

The target sample size was calculated based on the results of the previous study [14]. The primary outcome was BMD of the total anterior surface of the lumbar vertebrae, and the efficacy of the test food was evaluated by determining the difference in BMD between the time of screening to the completion of the 24 -week intervention period (post-24W). In the previous study, the mean change at post- $24 \mathrm{~W}$ was $0.005 \mathrm{~g} / \mathrm{cm}^{2}$ for the Test food group and $-0.017 \mathrm{~g} / \mathrm{cm}^{2}$ for the Placebo group, and the difference between the groups was $0.022 \mathrm{~g} / \mathrm{cm}^{2}$. The standard deviation was 0.019 . A significance level $(\alpha)$ of 0.05 and a power $(1-\beta)$ of 0.90 were set and it was finally determined that assigning approximately 17 subjects per group would be satisfactory. Thus, 19 subjects were included to account for dropout during the study period. Therefore, the target sample size was 34 subjects, and the actual sample size was 38 subjects in this study.

We decided on the sample size based on the BMD of the total anterior surface of the lumbar vertebrae, as described in a previous study [14]. 


\section{Selection, randomization, and blinding}

The 63 subjects who consented to participate in this study were determined by the principal investigator to be eligible for inclusion. The participants underwent bone density measurements when they were screened and tested, prior to ingesting the test substance. The results indicated that those with a total young adult mean (YAM) of $70-100 \%$ in the anterior surface of the lumbar vertebrae were eligible to participate. The lower limits of the BMD values for each individual were utilized. Then, to avoid major differences in age and mean \pm standard deviation (SD) BMD (total left femoral neck and anterior surface of the lumbar vertebrae), 19 participants were placed in the Test food group and another 19 in the Placebo group. Group allotments were conducted by an intermediary study controller using StatLight \#11 (Yukms Co., Ltd., Kanagawa, Japan). The allocation ratio for the groups was 1:1. The group allocation was unknown to the study participants, principal investigator, outcome assessors, and all other staff involved in this study; none of these individuals were involved in the allocation process.

\section{Test food}

The test food used in the intervention was corn syrup containing maltobionic acid (SourOligo, San-ei Sucrochemical Co., Ltd. Aichi, Japan). Corn syrup containing maltobionic acid comprises $40.3 \%$ maltobionic acid, $16.5 \%$ maltotronic acid, $13.2 \%$ other carbohydrates, and $30.0 \%$ moisture. The placebo food was a maltose syrup (contain 50.8\% maltose, $12.5 \%$ maltotriose, $6.7 \%$ other carbohydrates, and 30.0\% moisture; San-ei Sucrochemical Co., Ltd.). Prior to the start of the study, the institutional review board confirmed that the foods could not be distinguished based on odor or color. The study participants ingested $7 \mathrm{~g}$ per day after meals with cold water or warm water. The intervention period was 24 weeks.

\section{Outcome measures}

Examinations were conducted at baseline and at 12 and 24 weeks after initiating the intervention.

\section{Primary outcome: $X$-ray examination (dual-energy $X$-ray absorptiometry method)}

X-ray examinations consisted of dual-energy X-ray absorptiometry (DEXA) using a Discovery Xray BMD measuring device (Hologic Inc., USA) that was used to measure total lumbar vertebral anterior surface (L2-L4), bone area, bone mineral content (BMC), T-score, YAM value Z score prior to ingestion, 12 weeks post-intervention (post-12W), and $24 \mathrm{~W}$ post-intervention (post-24W) via medical scanning. The T-score refers to the value for which the index was specified using the mean BMD value (reference value) of young age as 0 and standard deviations as $1 \mathrm{SD}$. The YAM value represents the mean BMD of young adults (lumbar: $1.011 \mathrm{~g} / \mathrm{cm}^{2}$, femoral neck: $0.787 \mathrm{~g} / \mathrm{cm}^{2}$ ) as $100 \%$ and the bone density of the study participants as a percentage [15]. The Z-scores indicate values for which indices were specified using mean BMD values at the same ages as 0 and standard deviations as 1 SD. 


\section{Secondary outcome: Peripheral blood test}

Approximately $21 \mathrm{~mL}$ of venous blood and $13 \mathrm{~mL}$ of urine were collected from each study participant at Takara Clinic. The bone metabolism markers used included Tartrate-resistant acid phosphatase 5b (TRACP-5b), osteocalcin (OC), bone-specific alkaline phosphatase (BAP), type I collagen cross-linked N-telopeptides (u-NTx, s-NTx), and deoxypyridinoline (DPD). Measurements were conducted by LSI Medience Corporation (Tokyo, Japan).

\section{Safety assessment items}

\subsection{Physical measurements and physiological testing}

We measured height, weight, body mass index (BMI), body fat percentage, systolic blood pressure (BP), diastolic BP, and pulse. Height was measured only once following the conclusion of the participants' orientation meeting. The height measurement was conducted at ORTHOMEDICO Inc., while all other measurements were conducted at Takara Clinic.

\subsection{Peripheral blood test}

Approximately $15 \mathrm{~mL}$ of blood was collected from each study participant at Takara Clinic.

Hematological testing consisted of measurements of white blood count (WBC), red blood cell count (RBC), hemoglobin (Hb), hematocrit (Ht), platelet count (PLT), mean corpuscular volume (MCV), mean corpuscular hemoglobin $(\mathrm{MCH})$, mean corpuscular hemoglobin concentration (MCHC), and white blood cell imaging using a Sysmex XE-2100 (Sysmex Corporation, Hyogo, Japan), HEG-L (Sysmex Corporation), and an optical microscope BX41 (Olympus Corporation, Tokyo, Japan). Measurements were conducted by LSI Medience Corporation.

Blood biochemistry testing consisted of measurements of the following: aspartate transaminase (AST), alanine transaminase (ALT), $\gamma$-glutamyl transferase $(\gamma$-GT), alkaline phosphatase (ALP), lactate dehydrogenase (LD), leukocyte alkaline phosphatase (LAP), total bilirubin (T-Bil), direct bilirubin (D-Bil), indirect bilirubin (I-Bil), cholinesterase (ChE), zinc sulfate turbidity test (ZTT), total protein (TP), urea nitrogen (UN), creatinine (CRE), uric acid (UA), creatine kinase $(\mathrm{CK})$, sodium $(\mathrm{Na})$, potassium $(\mathrm{K})$, chlorine $(\mathrm{Cl})$, calcium $(\mathrm{Ca})$, inorganic phosphorus (IP), serum iron (Fe), serum amylase (AMY), total cholesterol (TC), high-density lipoprotein cholesterol (HDL-C), low-density lipoprotein cholesterol (LDL-C), triglycerides (TG), glucose (Glu), glycated hemoglobin (HbAlc), and glucoalbumin (GA). These measurements were performed using an H7700 (Hitachi High-Technologies Corporation, Tokyo, Japan) and a JCABM9130 (JEOL Ltd., Tokyo, Japan. Measurements were conducted by LSI Medience Corporation.

\subsection{Urinalysis}

Approximately $50 \mathrm{~mL}$ of urine was collected from each study participant, and protein (Pro), glucose (Glu), urobilinogen (Uro), bilirubin (Bil), ketone bodies (Ket), pH, and occult blood (Bld) were measured. Measurements were conducted by LSI Medience Corporation.

\section{Diet survey}

We conducted a questionnaire survey of the participants' nutritional intake for a 1-month period proximate to the study at pre-ingestion, post-12W, and post- $24 \mathrm{~W}$ using a brief-type selfadministered diet history questionnaire (BDHQ) $[16,17]$ that investigated the intake of 56 foods and beverages in the last month. Dietary intake in terms of energetics and selected nutrients was estimated based on computer algorithms. 


\section{Statistical analysis}

All outcomes in this study were examined using outpatient testing at the pre-ingestion, post-12W, and post-24W time points, and intra- and inter-group comparisons were made. The intra-group comparisons were performed using Dunnett's test with the time points and study participants as the fixed factors using the measured values in the following comparisons: pre-ingestion vs. post$12 \mathrm{~W}$ and pre-ingestion vs. post $24 \mathrm{~W}$. Inter-group comparisons were performed by determining the amount of change at all time points in the Test food group versus Placebo group. The amount of change was determined by subtracting the pre-ingestion measured value from the post- $12 \mathrm{~W}$ or post-24W measured value. The pre-ingestion measured value and amount of change were analyzed using Student's $t$-test.

All statistical analyses were performed using two-sided testing, and the standard of significance was set at 5\%. The software used was SPSS for Windows (ver. 23.0; IBM Japan, Tokyo, Japan) and Microsoft Excel 2007 and 2013 (Microsoft Japan, Tokyo, Japan). Redundancy with other time points or other items was given no consideration.

\section{RESULTS}

\section{Analysis of subjects}

Figure 1 shows a follow-up flow chart for the study participants. Of the 63 individuals who consented to participation in this study, 25 were excluded during interviews with the principal investigator or because of the inclusion/exclusion criteria. Finally, 38 individuals were enrolled in this study. We excluded 15 potential candidates: 1 subject who dropped out of the study, 3 subjects who violated compliance, and 4 subjects whose BMD of the total lumbar spine fell outside the 2SD range for any of the percentage changes, 2 who exhibited a percent change in serum total protein that exceeded the mean $\pm 2 \mathrm{SD}$ range, and 5 with abnormally high urinary NTx $(>89.0$ $\mathrm{nmol} \mathrm{BCE} / \mathrm{mmol} \cdot \mathrm{Cr}$ ). Among bone metabolism markers associated with osteoclasts, urinary NTx is the ultimate product of metabolism of bone during resorption and is positively correlated with decreased bone density [18]. The total protein in the blood is negatively correlated with bone density, and it seems to show the effect on the bone density [19]. Therefore, at the case review meeting after the completion of the study, we excluded 5 subjects who showed abnormally high values $(>89.0 \mathrm{nmol} \mathrm{BCE} / \mathrm{mmol} \cdot \mathrm{Cr}$ ) of urine NTx before and after ingestion of the test food and 2 subjects who showed an outlier value for any of the percentage changes in each test, from preingestion onward, for total protein in blood.

Thus, the final subject population consisted of per-protocol sets of 13 subjects in the Placebo group (mean age, $55.8 \pm 4.4$ years) and 10 subjects in the Test food group (mean age, $58.2 \pm 5.4$ years). The subject population for the safety assessment items consisted of study subjects who underwent at least one intervention. There were 17 subjects (mean age, $55.9 \pm 4.2$ years) in the Placebo group and 18 subjects in the Test food group (mean age, $57.3 \pm 4.6$ years). The results of the dietary survey by BDHQ showed no significant differences in nutrient intake between Test food group and Placebo group, and no study participants had an intake rate of less than $90 \%$. 


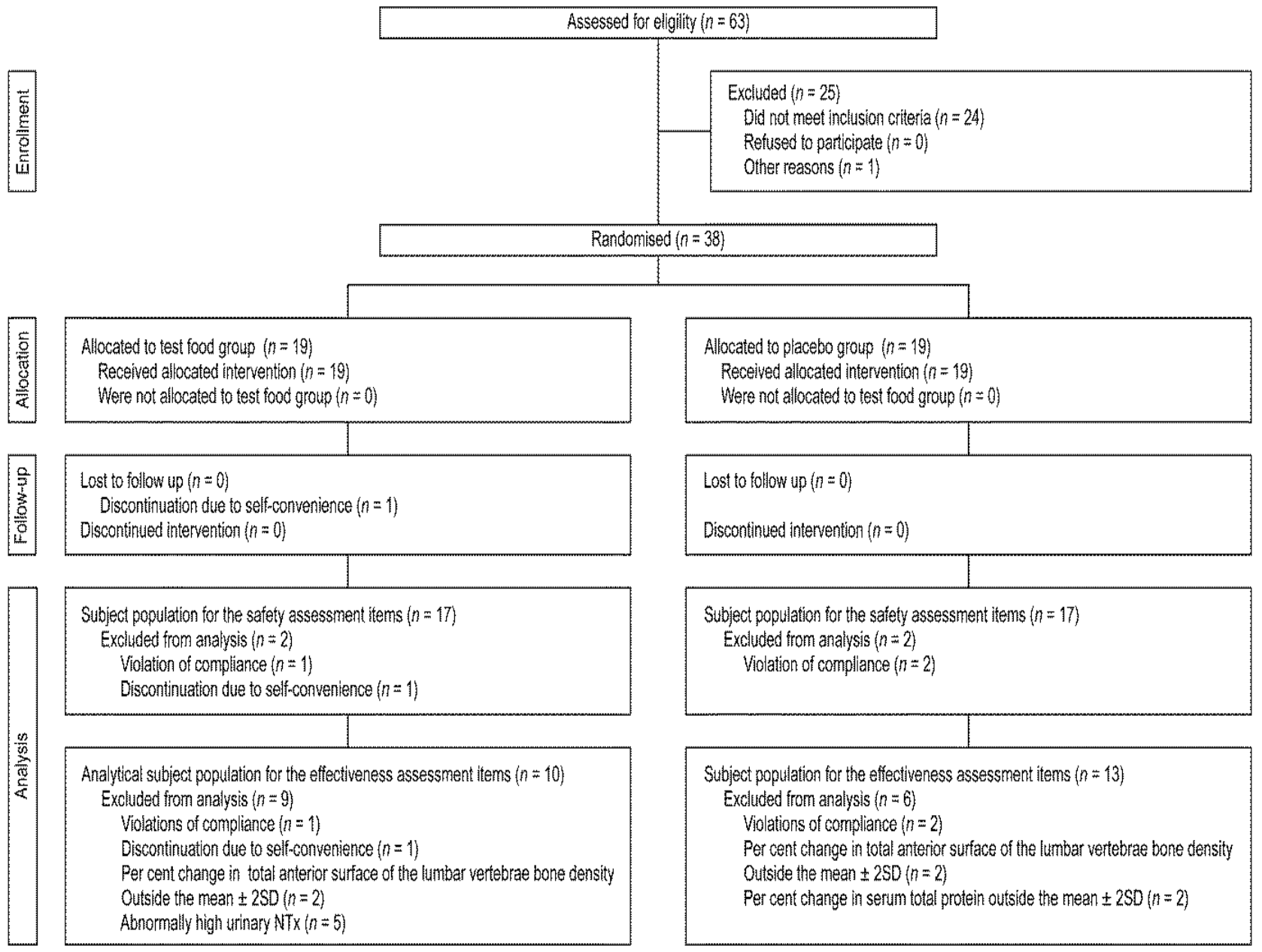

Figure 1. Follow-up flow chart for the study participants

\section{Efficacy assessment}

The background characteristics of the 23 subjects in the subject population for the efficacy analysis are shown in Table 1. The efficacy assessment items (BMD measurements for lumbar vertebrae anterior surface) are shown in Table 2. The peripheral blood test results are shown in Table 3.

Table 1. Characteristics of subjects

\begin{tabular}{|c|c|c|c|c|c|c|c|}
\hline \multirow[b]{2}{*}{ Age } & \multirow[b]{2}{*}{ years } & \multicolumn{3}{|c|}{$\begin{array}{l}\text { Test food group } \\
(n=10)\end{array}$} & \multicolumn{3}{|c|}{$\begin{array}{l}\text { Placebo group } \\
(n=13)\end{array}$} \\
\hline & & 58.2 & & 5.4 & 55.8 & \pm & 4.4 \\
\hline Body height & $\mathrm{Cm}$ & 158.5 & \pm & 4.8 & 156.0 & \pm & 5.0 \\
\hline Body weight & $\mathrm{Kg}$ & 56.5 & \pm & 9.3 & 52.9 & \pm & 8.8 \\
\hline BMI & $\mathrm{kg} / \mathrm{m}^{2}$ & 22.6 & \pm & 3.9 & 21.8 & \pm & 4.3 \\
\hline $\begin{array}{l}\text { Body fat } \\
\text { percentage }\end{array}$ & $\%$ & 27.7 & \pm & 7.8 & 26.4 & \pm & 5.8 \\
\hline $\mathrm{BP}$ & $\mathrm{mmHg}$ & 115.1 & \pm & 15.3 & 123.0 & \pm & 14.7 \\
\hline Diastolic BP & $\mathrm{mmHg}$ & 70.5 & \pm & 12.6 & 76.3 & \pm & 12.7 \\
\hline Pulse & Bpm & 78.6 & \pm & 7.3 & 75.3 & \pm & 8.6 \\
\hline
\end{tabular}

Values are mean $\pm \mathrm{SD}$ 
Table 2. Changes in anterior surface of lumbar vertebrae (L2, L3, L4, L2-L4)

\begin{tabular}{|c|c|c|c|c|c|c|c|c|c|c|c|c|c|c|c|c|c|c|c|}
\hline \multirow{4}{*}{ Bone area (L2) } & \multirow{4}{*}{$\mathrm{cm}^{2}$} & \multirow{3}{*}{$\begin{array}{l}\text { Group } \\
\text { Test food }\end{array}$} & \multirow{3}{*}{$\begin{array}{l}n \\
10 \\
\end{array}$} & \multirow{2}{*}{\multicolumn{3}{|c|}{ Pre-ingestion }} & \multirow{2}{*}{\multicolumn{3}{|c|}{ Post-12W }} & \multirow{2}{*}{\multicolumn{3}{|c|}{ Post-24W }} & \multirow{2}{*}{\multicolumn{3}{|c|}{$\begin{array}{l}\text { Pre-ingestion vs. Post-12W } \\
\text { Amount of change }\end{array}$}} & \multirow{2}{*}{\multicolumn{4}{|c|}{$\begin{array}{l}\text { Pre-ingestion vs. Post-24W } \\
\text { Amount of change }\end{array}$}} \\
\hline & & & & & & & & & & & & & & & & & & & \\
\hline & & & & 13.34 & \pm & 1.07 & 13.65 & \pm & 0.97 & 13.53 & \pm & 0.92 & 0.31 & \pm & 0.44 & 0.19 & \pm & 0.48 & \# \\
\hline & & Placebo & 13 & 13.79 & \pm & 0.94 & 13.76 & \pm & 1.00 & 13.47 & \pm & 0.86 & -0.03 & \pm & 0.49 & -0.33 & \pm & 0.65 & \\
\hline \multirow{2}{*}{ BMC (L2) } & \multirow{2}{*}{ g } & Test food & 10 & 11.09 & \pm & 1.44 & 11.11 & \pm & 1.64 & 11.21 & \pm & 1.55 & 0.02 & \pm & 0.38 & 0.13 & \pm & 0.46 & $\#$ \\
\hline & & Placebo & 13 & 11.88 & \pm & 1.62 & 11.76 & \pm & 1.87 & 11.36 & \pm & 1.73 & -0.12 & \pm & 0.55 & -0.52 & \pm & 0.73 & \\
\hline \multirow{2}{*}{ BMD (L2) } & \multirow{2}{*}{$\mathrm{g} / \mathrm{cm}^{2}$} & Test food & 10 & 0.83 & \pm & 0.10 & 0.81 & \pm & 0.11 & 0.83 & \pm & 0.10 & -0.02 & \pm & 0.02 & 0.00 & \pm & 0.02 & \\
\hline & & Placebo & 13 & 0.86 & \pm & 0.09 & 0.85 & \pm & 0.09 & 0.84 & \pm & 0.10 & -0.01 & \pm & 0.02 & -0.02 & \pm & 0.03 & \\
\hline \multirow{2}{*}{ T-score (L2) } & & Test food & 10 & -1.78 & \pm & 0.93 & -1.95 & \pm & 0.99 & -1.82 & \pm & 0.94 & -0.17 & \pm & 0.23 & -0.04 & \pm & 0.16 & \\
\hline & & Placebo & 13 & -1.50 & \pm & 0.84 & -1.58 & \pm & 0.84 & -1.68 & \pm & 0.98 & -0.08 & \pm & 0.20 & -0.18 & \pm & 0.32 & \\
\hline \multirow{2}{*}{ YAM value (L2) } & \multirow{2}{*}{$\%$} & Test food & 10 & 81.70 & \pm & 9.66 & 79.90 & \pm & 10.45 & 81.40 & \pm & 9.94 & -1.80 & \pm & 2.39 & -0.30 & \pm & 1.95 & \\
\hline & & Placebo & 13 & 84.31 & \pm & 8.72 & 83.54 & \pm & 8.75 & 82.69 & \pm & 10.23 & -0.77 & \pm & 2.13 & -1.62 & \pm & 3.25 & \\
\hline \multirow{2}{*}{ Z score (L2) } & \multirow{2}{*}{-} & Test food & 10 & 1.09 & \pm & 1.02 & 0.99 & \pm & 1.02 & 1.15 & \pm & 1.00 & -0.10 & \pm & 0.24 & 0.06 & \pm & 0.16 & \\
\hline & & Placebo & 13 & 1.08 & \pm & 0.69 & 1.02 & \pm & 0.73 & 0.97 & \pm & 0.87 & -0.06 & \pm & 0.21 & -0.12 & \pm & 0.30 & \\
\hline \multirow{2}{*}{ Bone area (L3) } & \multirow{2}{*}{$\mathrm{cm}^{2}$} & Test food & 10 & 14.97 & \pm & 1.18 & 14.97 & \pm & 1.07 & 15.11 & \pm & 1.14 & 0.00 & \pm & 0.49 & 0.14 & \pm & 0.49 & \\
\hline & & Placebo & 13 & 14.60 & \pm & 0.93 & 14.53 & \pm & 0.96 & 14.55 & \pm & 0.93 & -0.07 & \pm & 0.47 & -0.04 & \pm & 0.40 & \\
\hline \multirow{2}{*}{ BMC (L3) } & \multirow{2}{*}{$\mathrm{g}$} & Test food & 10 & 12.54 & \pm & 1.78 & 12.51 & \pm & 1.49 & 12.76 & \pm & 1.70 & -0.03 & \pm & 0.66 & 0.22 & \pm & 0.42 & $\#$ \\
\hline & & Placebo & 13 & 12.94 & \pm & 1.79 & 12.74 & \pm & 1.91 & 12.66 & \pm & 2.00 & -0.20 & \pm & 0.66 & -0.28 & \pm & 0.64 & \\
\hline \multirow{2}{*}{ BMD (L3) } & \multirow{2}{*}{$\mathrm{g} / \mathrm{cm}^{2}$} & Test food & 10 & 0.84 & \pm & 0.09 & 0.84 & \pm & 0.09 & 0.85 & \pm & 0.10 & 0.00 & \pm & 0.02 & 0.01 & \pm & 0.02 & \\
\hline & & Placebo & 13 & 0.89 & \pm & 0.11 & 0.88 & \pm & 0.10 & 0.87 & \pm & 0.11 & -0.01 & \pm & 0.02 & -0.02 & \pm & 0.03 & \\
\hline \multirow{2}{*}{ T-score (L3) } & 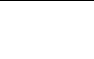 & Test food & 10 & -1.92 & \pm & 0.86 & -1.93 & \pm & 0.81 & -1.84 & \pm & 0.92 & -0.01 & \pm & 0.19 & 0.08 & \pm & 0.15 & $\#$ \\
\hline & - & Placebo & 13 & -1.48 & \pm & 0.97 & -1.57 & \pm & 0.95 & -1.64 & \pm & 1.03 & -0.09 & \pm & 0.22 & -0.16 & \pm & 0.26 & \\
\hline YAM value (I 3) & 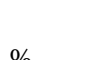 & Test food & 10 & 80.10 & \pm & 9.09 & 80.00 & \pm & 8.52 & 80.80 & \pm & 9.46 & -0.10 & \pm & 2.28 & 0.70 & \pm & 1.57 & $\#$ \\
\hline Y AM value (LS) & $\%$ & Placebo & 13 & 84.62 & \pm & 10.16 & 83.62 & \pm & 9.84 & 82.92 & \pm & 10.76 & -1.00 & \pm & 2.31 & -1.69 & \pm & 2.63 & \\
\hline & 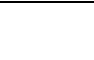 & Test food & 10 & 0.70 & \pm & 0.97 & 0.74 & \pm & 0.92 & 0.85 & \pm & 1.01 & 0.04 & \pm & 0.20 & 0.15 & \pm & 0.16 & $\#$ \\
\hline & - & Placebo & 13 & 0.84 & \pm & 0.89 & 0.78 & \pm & 0.87 & 0.75 & \pm & 0.95 & -0.05 & \pm & 0.24 & -0.09 & \pm & 0.28 & \\
\hline
\end{tabular}




\begin{tabular}{|c|c|c|c|c|c|c|c|c|c|c|c|c|c|c|c|c|c|c|c|}
\hline \multirow{4}{*}{ Bone area (L4) } & \multirow{4}{*}{$\mathrm{cm}^{2}$} & \multirow{3}{*}{$\begin{array}{l}\text { Group } \\
\text { Test food }\end{array}$} & \multirow{3}{*}{$\begin{array}{l}n \\
10\end{array}$} & \multirow{2}{*}{\multicolumn{3}{|c|}{ Pre-ingestion }} & \multirow{2}{*}{\multicolumn{3}{|c|}{ Post-12W }} & \multirow{2}{*}{\multicolumn{3}{|c|}{ Post-24W }} & \multirow{2}{*}{\multicolumn{3}{|c|}{$\begin{array}{l}\text { Pre-ingestion vs. Post-12W } \\
\text { Amount of change }\end{array}$}} & \multirow{2}{*}{\multicolumn{4}{|c|}{$\begin{array}{l}\text { Pre-ingestion vs. Post-24W } \\
\text { Amount of change }\end{array}$}} \\
\hline & & & & & & & & & & & & & & & & & & & \\
\hline & & & & 14.74 & \pm & 2.55 & 15.77 & \pm & 2.25 & 15.73 & \pm & 2.45 & 1.03 & \pm & 2.63 & 0.99 & \pm & 2.72 & \\
\hline & & Placebo & 13 & 15.70 & \pm & 1.41 & 15.79 & \pm & 1.18 & 15.68 & \pm & 1.20 & 0.09 & \pm & 0.81 & -0.01 & \pm & 0.64 & \\
\hline \multirow{2}{*}{ BMC (L4) } & \multirow{2}{*}{$\mathrm{g}$} & Test food & 10 & 12.57 & \pm & 2.24 & 13.19 & \pm & 1.86 & 13.37 & \pm & 1.97 & 0.62 & \pm & 1.88 & 0.80 & \pm & 1.93 & \\
\hline & & Placebo & 13 & 13.62 & \pm & 2.16 & 13.63 & \pm & 1.73 & 13.57 & \pm & 2.01 & 0.02 & \pm & 1.07 & -0.05 & \pm & 0.58 & \\
\hline \multirow{2}{*}{ BMD (L4) } & \multirow{2}{*}{$\mathrm{g} / \mathrm{cm}^{2}$} & Test food & 10 & 0.86 & \pm & 0.08 & 0.84 & \pm & 0.10 & 0.85 & \pm & 0.08 & -0.01 & \pm & 0.04 & 0.00 & \pm & 0.03 & \\
\hline & & Placebo & 13 & 0.87 & \pm & 0.11 & 0.86 & \pm & 0.10 & 0.86 & \pm & 0.11 & 0.00 & \pm & 0.04 & 0.00 & \pm & 0.02 & \\
\hline \multirow{2}{*}{ T-score (L4) } & \multirow{2}{*}{-} & Test food & 10 & -1.72 & \pm & 0.73 & -1.85 & \pm & 0.84 & -1.73 & \pm & 0.68 & -0.13 & \pm & 0.32 & -0.01 & \pm & 0.24 & \\
\hline & & Placebo & 13 & -1.61 & \pm & 0.99 & -1.63 & \pm & 0.87 & -1.65 & \pm & 0.95 & -0.02 & \pm & 0.33 & -0.05 & \pm & 0.20 & \\
\hline \multirow{2}{*}{ YAM value (L4) } & \multirow{2}{*}{$\%$} & Test food & 10 & 81.30 & \pm & 7.67 & 80.00 & \pm & 9.19 & 81.20 & \pm & 7.51 & -1.30 & \pm & 3.47 & -0.10 & \pm & 2.56 & \\
\hline & & Placebo & 13 & 82.31 & \pm & 10.76 & 82.08 & \pm & 9.55 & 82.23 & \pm & 10.20 & -0.23 & \pm & 3.49 & -0.08 & \pm & 2.22 & \\
\hline \multirow{2}{*}{ Z score (L4) } & \multirow{2}{*}{-} & Test food & 10 & 0.36 & \pm & 0.83 & 0.26 & \pm & 0.90 & 0.38 & \pm & 0.85 & -0.10 & \pm & 0.32 & 0.02 & \pm & 0.26 & \\
\hline & & Placebo & 13 & 0.18 & \pm & 0.96 & 0.21 & \pm & 0.87 & 0.23 & \pm & 0.94 & 0.02 & \pm & 0.33 & 0.05 & \pm & 0.21 & \\
\hline \multirow{2}{*}{ Bone area (L2-4) } & \multirow{2}{*}{$\mathrm{cm}^{2}$} & Test food & 10 & 43.05 & \pm & 3.81 & 44.39 & \pm & 3.55 & 44.37 & \pm & 4.01 & 1.34 & \pm & 2.44 & 1.31 & \pm & 3.13 & \\
\hline & & Placebo & 13 & 44.09 & \pm & 2.50 & 44.08 & \pm & 2.62 & 43.70 & \pm & 2.61 & -0.01 & \pm & 0.89 & -0.39 & \pm & 1.00 & \\
\hline \multirow{2}{*}{ BMC (L2-4) } & \multirow{2}{*}{$\mathrm{g}$} & Test food & 10 & 36.50 & \pm & 4.54 & 36.81 & \pm & 4.56 & 37.35 & \pm & 4.52 & 0.31 & \pm & 2.37 & 0.85 & \pm & 2.64 & $\#$ \\
\hline & & Placebo & 13 & 38.44 & \pm & 4.99 & 38.13 & \pm & 5.09 & 37.58 & \pm & 5.41 & -0.30 & \pm & 1.07 & -0.86 & \pm & 1.15 & \\
\hline \multirow{2}{*}{ BMD (L2-4) } & \multirow{2}{*}{$\mathrm{g} / \mathrm{cm}^{2}$} & Test food & 10 & 0.84 & \pm & 0.09 & 0.83 & \pm & 0.09 & 0.84 & \pm & 0.08 & -0.01 & \pm & 0.02 & 0.00 & \pm & 0.01 & $\#$ \\
\hline & & Placebo & 13 & 0.87 & \pm & 0.10 & 0.86 & \pm & 0.09 & 0.86 & \pm & 0.10 & -0.01 & \pm & 0.02 & -0.01 & \pm & 0.02 & \\
\hline \multirow{2}{*}{ T-score (L2-4) } & 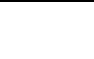 & Test food & 10 & -1.44 & \pm & 0.72 & -1.52 & \pm & 0.76 & -1.43 & \pm & 0.70 & -0.08 & \pm & 0.13 & 0.01 & \pm & 0.07 & \\
\hline & - & Placebo & 13 & -1.17 & \pm & 0.81 & -1.22 & \pm & 0.78 & -1.27 & \pm & 0.85 & -0.05 & \pm & 0.15 & -0.10 & \pm & 0.16 & \\
\hline YAM & 0 & Test food & 10 & 83.30 & \pm & 8.60 & 82.10 & \pm & 9.05 & 83.40 & \pm & 8.38 & -1.20 & \pm & 1.48 & 0.10 & \pm & 0.99 & $\#$ \\
\hline Y AIM value (LL-4) & $\%$ & Placebo & 13 & 86.23 & \pm & 9.55 & 85.46 & \pm & 9.17 & 84.77 & \pm & 10.16 & -0.77 & \pm & 1.48 & -1.46 & \pm & 1.71 & \\
\hline 2-4) & 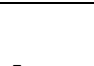 & Test food & 10 & 0.00 & \pm & 0.67 & -0.08 & \pm & 0.65 & 0.05 & \pm & 0.65 & -0.08 & \pm & 0.11 & 0.05 & \pm & 0.08 & \\
\hline Z score (L L-4) & - & Placebo & 13 & 0.00 & \pm & 0.64 & -0.03 & \pm & 0.62 & -0.02 & \pm & 0.70 & -0.03 & \pm & 0.14 & -0.02 & \pm & 0.13 & \\
\hline
\end{tabular}

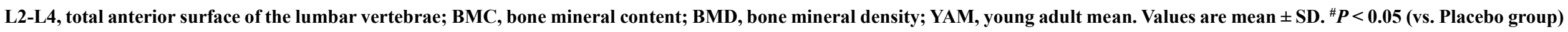


Table 3. Changes in bone metabolism markers

\begin{tabular}{|c|c|c|c|c|c|c|c|c|c|c|c|c|c|c|c|c|c|c|}
\hline \multirow{4}{*}{ TRACP-5b } & \multirow{4}{*}{$\mathrm{mU} / \mathrm{dL}$} & \multirow{3}{*}{$\begin{array}{l}\text { Group } \\
\text { Test food }\end{array}$} & \multirow{3}{*}{$\begin{array}{l}n \\
10\end{array}$} & \multirow{2}{*}{\multicolumn{3}{|c|}{ Pre-ingestion }} & \multirow{2}{*}{\multicolumn{3}{|c|}{ Post-12W }} & \multirow{2}{*}{\multicolumn{3}{|c|}{ Post-24W }} & \multirow{2}{*}{\multicolumn{3}{|c|}{$\begin{array}{l}\text { Pre-ingestion vs. Post-12W } \\
\text { Amount of change }\end{array}$}} & \multirow{2}{*}{\multicolumn{3}{|c|}{$\begin{array}{l}\text { Pre-ingestion vs. Post-24W } \\
\text { Amount of change }\end{array}$}} \\
\hline & & & & & & & & & & & & & & & & & & \\
\hline & & & & 439.20 & \pm & 105.20 & 378.90 & \pm & 83.90 & 359.30 & \pm & 60.10 & -60.30 & \pm & 65.90 & -79.90 & \pm & 74.70 \\
\hline & & Placebo & 13 & 447.50 & \pm & 127.20 & 372.00 & \pm & 104.50 & 386.10 & \pm & 126.10 & -75.50 & \pm & 100.50 & -61.40 & \pm & 59.50 \\
\hline \multirow{2}{*}{$\mathrm{OC}$} & \multirow{2}{*}{$\mathrm{ng} / \mathrm{mL}$} & Test food & 10 & 15.00 & \pm & 5.30 & 18.20 & \pm & 9.30 & 15.70 & \pm & 6.60 & 3.20 & \pm & 4.80 & 0.60 & \pm & 2.10 \\
\hline & & Placebo & 13 & 16.00 & \pm & 4.50 & 17.40 & \pm & 5.20 & 17.30 & \pm & 6.40 & 1.40 & \pm & 2.50 & 1.30 & \pm & 3.80 \\
\hline \multirow{2}{*}{ BAP } & \multirow{2}{*}{$\mu \mathrm{g} / \mathrm{L}$} & Test food & 10 & 13.60 & \pm & 3.40 & 12.80 & \pm & 3.00 & 13.50 & \pm & 2.10 & -0.80 & \pm & 2.20 & -0.10 & \pm & 1.80 \\
\hline & & Placebo & 13 & 13.20 & \pm & 3.40 & 12.80 & \pm & 3.30 & 13.90 & \pm & 5.10 & -0.50 & \pm & 1.20 & 0.60 & \pm & 3.20 \\
\hline \multirow{2}{*}{ s-NTx } & \multirow{2}{*}{ nmol BCE/L } & Test food & 10 & 16.90 & \pm & 6.60 & 17.90 & \pm & 6.30 & 15.80 & \pm & 4.20 & 1.00 & \pm & 2.10 & -1.10 & \pm & 3.60 \\
\hline & & Placebo & 13 & 16.70 & \pm & 3.50 & 17.00 & \pm & 4.00 & 17.50 & \pm & 4.50 & 0.30 & \pm & 3.30 & 0.80 & \pm & 3.10 \\
\hline \multirow{2}{*}{ u-NTx } & \multirow{2}{*}{$\mathrm{nmol} \mathrm{BCE} / \mathrm{mmol} \cdot \mathrm{Cr}$} & Test food & 10 & 46.70 & \pm & 14.90 & 42.10 & \pm & 16.10 & 47.90 & \pm & 17.40 & -4.60 & \pm & 16.10 & 1.20 & \pm & 21.60 \\
\hline & & Placebo & 13 & 52.00 & \pm & 12.80 & 52.90 & \pm & 18.40 & 53.40 & \pm & 16.40 & 0.90 & \pm & 15.80 & 1.40 & \pm & 16.50 \\
\hline \multirow{2}{*}{ DPD } & \multirow{2}{*}{$\mathrm{nmol} / \mathrm{mmol} \cdot \mathrm{Cr}$} & Test food & 10 & 5.50 & \pm & 0.90 & 5.60 & \pm & 0.90 & 5.40 & \pm & 0.80 & 0.10 & \pm & 0.80 & -0.10 & \pm & 1.00 \\
\hline & & Placebo & 13 & 6.50 & \pm & 2.40 & 7.00 & \pm & 2.40 & 7.10 & \pm & 3.50 & 0.50 & \pm & 1.60 & 0.70 & \pm & 2.40 \\
\hline
\end{tabular}

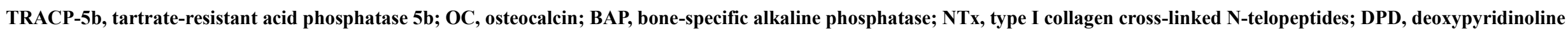
Values are mean $\pm \mathrm{SD} ;{ }^{\sharp} \boldsymbol{P}<0.05$ (vs. Placebo group) 


\section{Anterior surface of lumbar vertebrae}

Investigation of the amount of change indicated that at post-24W, the bone area of L2 (Test food: $+0.19 \pm 0.48 \mathrm{~cm}^{2}$, Placebo: $-0.33 \pm 0.65 \mathrm{~cm}^{2}: P=0.048$ ), BMC of L2 (Test food: $+0.13 \pm 0.46 \mathrm{~g}$, Placebo: $-0.52 \pm 0.73 \mathrm{~g}: P=0.023$ ), BMC of L3 (Test food: $+0.22 \pm 0.42 \mathrm{~g}$, Placebo: $-0.28 \pm 0.64$ g: $P=0.042$ ), BMD of L3 (Test food: $+0.08 \pm 0.017 \mathrm{~g} / \mathrm{cm}^{2}$, Placebo: $-0.018 \pm 0.027 \mathrm{~g} / \mathrm{cm}^{2}: P=$ 0.016), T-score of L3 (Test food: $+0.08 \pm 0.15$, Placebo: $-0.16 \pm 0.26: P=0.017$ ), YAM of L3 (Test food: $+0.70 \pm 1.57 \%$, Placebo: $-1.69 \pm 2.63: P=0.019$ ), Z-score of L3 (Test food: $+0.15 \pm$ 0.16 , Placebo: $-0.09 \pm 0.28: P=0.024)$, BMC of the total anterior surface of the lumbar vertebrae (Test food: $+0.85 \pm 2.64 \mathrm{~g}$, Placebo: $-0.86 \pm 1.15 \mathrm{~g}: P=0.048$ ), BMD of the total anterior surface of the lumbar vertebrae (Test food: $+0.002 \pm 0.009 \mathrm{~g} / \mathrm{cm}^{2}$, Placebo: $-0.013 \pm 0.018 \mathrm{~g} / \mathrm{cm}^{2}: P=$ 0.033), and YAM of the total anterior surface of the lumbar vertebrae (Test food: $+0.10 \pm 0.99 \%$, Placebo: $-1.46 \pm 1.71 \%: P=0.018$ ) in the Test food group were significantly higher than in the Placebo group.

\section{Peripheral blood test (bone metabolism markers)}

Although there were no significant differences in urinary DPD, values tended to be consistently lower in the Test food group than in the Placebo group from pre-intake to 24 weeks post-intake.

\section{Safety assessment}

The physical measurements and physiological test results for all subjects are presented in Table 4. The peripheral blood test results and urinalysis results are presented in Tables 5 and 6, respectively. The results of the physical measurements, physiological tests, and peripheral blood tests indicate that, from pre-ingestion to post- $24 \mathrm{~W}$, the values were within the normal range or slightly outside the normal range. The urinalysis results indicated that the items for which values were outside standard values at post-12W included Pro (in 1 subject) in the Test food group versus included Pro (in 1), and Bld (in 1) in the Placebo group. At post-24W, values were outside the standard range for Pro (in 2), and Bld (in 1) in the Test food group versus Bld (in 2) in the Placebo group. 
Table 4. Physical parameters (safety assessment)

\begin{tabular}{|c|c|c|c|c|c|c|c|c|c|c|c|c|c|}
\hline & & Group & $n$ & Pre-in & ion & & & Post-12W & & & Post- & & \\
\hline \multirow{2}{*}{ Age } & \multirow{2}{*}{ Years } & Test food & 17 & 57.3 & \pm & 4.6 & & & & & & & \\
\hline & & Placebo & 17 & 55.9 & \pm & 4.2 & & & & & & & \\
\hline \multirow{2}{*}{ Body height } & \multirow{2}{*}{$\mathrm{Cm}$} & Test food & 17 & 159.8 & \pm & 4.3 & $\#$ & & & & & & \\
\hline & & Placebo & 17 & 156.0 & \pm & 5.6 & & & & & & & \\
\hline \multirow{2}{*}{ Body weight } & \multirow{2}{*}{$\mathrm{Kg}$} & Test food & 17 & 56.5 & \pm & 9.6 & & 55.9 & \pm & 10.0 & 56.3 & \pm & 10.1 \\
\hline & & Placebo & 17 & 52.8 & \pm & 8.3 & & 52.4 & \pm & 7.8 & 53.1 & \pm & 7.6 \\
\hline \multirow{2}{*}{ BMI } & \multirow{2}{*}{$\mathrm{kg} / \mathrm{m}^{2}$} & Test food & 17 & 22.2 & \pm & 3.8 & & 21.9 & \pm & 4.0 & 22.1 & \pm & 4.0 \\
\hline & & Placebo & 17 & 21.8 & \pm & 4.1 & & 21.7 & \pm & 3.9 & 21.9 & \pm & 3.9 \\
\hline \multirow{2}{*}{ Body fat percentage } & \multirow{2}{*}{$\%$} & Test food & 17 & 26.2 & \pm & 7.6 & & 26.6 & \pm & 7.0 & 26.0 & \pm & 7.7 \\
\hline & & Placebo & 17 & 26.2 & \pm & 6.1 & & 26.2 & \pm & 5.6 & 25.9 & \pm & 5.6 \\
\hline \multirow{2}{*}{ Systolic blood pressure } & \multirow{2}{*}{$\mathrm{mmHg}$} & Test food & 17 & 119.5 & \pm & 17.4 & & 118.7 & \pm & 22.1 & 118.4 & \pm & 14.9 \\
\hline & & Placebo & 17 & 120.7 & \pm & 15.2 & & 116.5 & \pm & 16.0 & 117.4 & \pm & 11.7 \\
\hline \multirow{2}{*}{ Diastolic blood pressure } & \multirow{2}{*}{$\mathrm{mmHg}$} & Test food & 17 & 74.2 & \pm & 13.6 & & 73.3 & \pm & 14.4 & 74.2 & \pm & 10.9 \\
\hline & & Placebo & 17 & 75.1 & \pm & 11.8 & & 74.3 & \pm & 10.4 & 72.4 & \pm & 7.7 \\
\hline \multirow{2}{*}{ Pulse rate } & \multirow{2}{*}{ Bpm } & Test food & 17 & 76.5 & \pm & 8.4 & & 73.4 & \pm & 8.8 & 74.2 & \pm & 9.2 \\
\hline & & Placebo & 17 & 73.6 & \pm & 9.2 & & 72.2 & \pm & 5.6 & 71.4 & \pm & 5.8 \\
\hline
\end{tabular}

\footnotetext{
Values are mean $\pm \mathrm{SD} ;{ }^{\#} P<0.05$ (vs. Placebo group)
} 
Table 5. Peripheral blood test (safety assessment) results

\begin{tabular}{|c|c|c|c|c|c|c|c|c|c|c|c|c|c|}
\hline \multirow{3}{*}{ WBC } & \multicolumn{2}{|c|}{ Reference value } & \multirow{2}{*}{$\begin{array}{l}\text { Group } \\
\text { Test food }\end{array}$} & \multirow{2}{*}{$\begin{array}{l}n \\
17\end{array}$} & \multicolumn{3}{|c|}{ Pre-ingestion } & \multicolumn{3}{|c|}{ Post-12W } & \multicolumn{3}{|c|}{ Post-24W } \\
\hline & 22000000 & tut & & & 5388.2 & \pm & 1540.7 & 5411.8 & \pm & 1311.4 & 5311.8 & \pm & 1251.4 \\
\hline & $3300-9000$ & $\mu \mathrm{L}$ & Placebo & 17 & 4735.3 & \pm & 926.0 & 4758.8 & \pm & 1308.7 & 4658.8 & \pm & 993.8 \\
\hline \multirow{2}{*}{$\mathrm{RBC}$} & \multirow{2}{*}{$380-500$} & \multirow{2}{*}{$\times 10^{4} / \mu \mathrm{L}$} & Test food & 17 & 450.0 & \pm & 27.0 & 442.1 & \pm & 25.4 & 452.4 & \pm & 26.2 \\
\hline & & & Placebo & 17 & 447.9 & \pm & 24.1 & 439.0 & \pm & 23.4 & 441.6 & \pm & 31.5 \\
\hline \multirow{2}{*}{$\mathrm{Hb}$} & \multirow{2}{*}{$11.5-15.0$} & \multirow{2}{*}{$\mathrm{g} / \mathrm{dL}$} & Test food & 17 & 13.6 & \pm & 0.9 & 13.3 & \pm & 0.9 & 13.7 & \pm & 0.8 \\
\hline & & & Placebo & 17 & 13.4 & \pm & 1.2 & 13.2 & \pm & 1.0 & 13.4 & \pm & 1.2 \\
\hline \multirow{2}{*}{$\mathrm{Ht}$} & \multirow{2}{*}{$34.8-45.0$} & \multirow{2}{*}{$\%$} & Test food & 17 & 43.0 & \pm & 2.8 & 42.3 & \pm & 2.2 & 43.4 & \pm & 2.5 \\
\hline & & & Placebo & 17 & 42.1 & \pm & 3.4 & 41.7 & \pm & 2.7 & 41.9 & \pm & 3.2 \\
\hline \multirow{2}{*}{ PLT } & \multirow{2}{*}{$14.0-34.0$} & \multirow{2}{*}{$\times 10^{4} / \mu \mathrm{L}$} & Test food & 17 & 25.1 & \pm & 5.9 & 24.1 & \pm & 5.2 & 25.1 & \pm & 5.0 \\
\hline & & & Placebo & 17 & 25.8 & \pm & 5.9 & 25.7 & \pm & 7.5 & 25.1 & \pm & 4.6 \\
\hline \multirow{2}{*}{$\mathrm{MCV}$} & \multirow{2}{*}{ 85-102 } & \multirow{2}{*}{$\mathrm{fL}$} & Test food & 17 & 95.6 & \pm & 3.0 & 95.6 & \pm & 2.9 & 96.1 & \pm & 3.4 \\
\hline & & & Placebo & 17 & 94.0 & & 5.6 & 94.9 & \pm & 5.2 & 94.8 & \pm & 4.5 \\
\hline \multirow{2}{*}{$\mathrm{MCH}$} & \multirow{2}{*}{$28.0-34.0$} & \multirow{2}{*}{$\mathrm{pg}$} & Test food & 17 & 30.2 & \pm & 1.1 & 30.1 & \pm & 1.2 & 30.3 & \pm & 1.2 \\
\hline & & & Placebo & 17 & 29.9 & \pm & 2.0 & 30.1 & \pm & 1.7 & 30.4 & \pm & 1.7 \\
\hline \multirow{2}{*}{$\mathrm{MCHC}$} & \multirow{2}{*}{$30.2-35.1$} & \multirow{2}{*}{$\%$} & Test food & 17 & 31.7 & \pm & 0.9 & 31.5 & \pm & 0.8 & 31.5 & \pm & 1.1 \\
\hline & & & Placebo & 17 & 31.8 & \pm & 1.1 & 31.7 & \pm & 0.8 & 32.0 & \pm & 1.0 \\
\hline \multirow{2}{*}{$\mathrm{Neu}$} & \multirow{2}{*}{$40.0-75.0$} & \multirow{2}{*}{$\%$} & Test food & 17 & 56.5 & \pm & 8.4 & 57.5 & \pm & 7.2 & 57.1 & \pm & 6.0 \\
\hline & & & Placebo & 17 & 57.8 & \pm & 7.7 & 56.5 & \pm & 6.4 & 57.1 & \pm & 7.8 \\
\hline Ivm & $180-490$ & $\%$ & Test food & 17 & 36.6 & \pm & 7.7 & 35.3 & \pm & 6.8 & 35.3 & \pm & 5.3 \\
\hline Буп & $10.0-49.0$ & 70 & Placebo & 17 & 35.3 & \pm & 7.4 & 35.9 & \pm & 6.6 & 35.6 & \pm & 7.1 \\
\hline Mon & $20-100$ & $\%$ & Test food & 17 & 4.3 & \pm & 1.1 & 4.6 & \pm & 0.9 & 4.8 & \pm & 1.0 \\
\hline IVIOn & $2.0-10.0$ & $\%$ & Placebo & 17 & 4.2 & \pm & 1.4 & 4.5 & \pm & 0.9 & 4.6 & \pm & 1.1 \\
\hline
\end{tabular}




\begin{tabular}{|c|c|c|c|c|c|c|c|c|c|c|c|c|c|}
\hline \multirow{3}{*}{ Eos } & \multicolumn{2}{|c|}{ Reference value } & \multirow{2}{*}{$\begin{array}{l}\text { Group } \\
\text { Test food }\end{array}$} & \multirow{2}{*}{$\begin{array}{l}n \\
17\end{array}$} & \multicolumn{3}{|c|}{ Pre-ingestion } & \multicolumn{3}{|c|}{ Post-12W } & \multicolumn{3}{|c|}{ Post-24W } \\
\hline & 00 ○ & 0 & & & 2.0 & \pm & 1.2 & 1.9 & \pm & 1.2 & 2.2 & \pm & 1.6 \\
\hline & $0.0-8.0$ & $\%$ & Placebo & 17 & 2.1 & \pm & 1.0 & 2.3 & \pm & 1.1 & 2.1 & \pm & 1.4 \\
\hline \multirow{2}{*}{ Bas } & \multirow{2}{*}{$0.0-2.0$} & \multirow{2}{*}{$\%$} & Test food & 17 & 0.6 & \pm & 0.3 & 0.6 & \pm & 0.3 & 0.6 & \pm & 0.4 \\
\hline & & & Placebo & 17 & 0.6 & \pm & 0.4 & 0.7 & \pm & 0.4 & 0.7 & \pm & 0.4 \\
\hline \multirow{2}{*}{ Neu level } & & \multirow{2}{*}{$/ \mu \mathrm{L}$} & Test food & 17 & 3113.3 & \pm & 1176.1 & 3169.9 & \pm & 1029.9 & 3063.7 & \pm & 893.2 \\
\hline & & & Placebo & 17 & 2757.7 & \pm & 775.0 & 2723.8 & \pm & 955.5 & 2699.4 & \pm & 891.6 \\
\hline \multirow{2}{*}{ Lym level } & & \multirow{2}{*}{$/ \mu \mathrm{L}$} & Test food & 17 & 1916.6 & \pm & 557.1 & 1864.4 & \pm & 413.2 & 1857.1 & \pm & 468.7 \\
\hline & & & Placebo & 17 & 1646.4 & \pm & 394.6 & 1672.3 & \pm & 485.2 & 1619.0 & \pm & 353.5 \\
\hline \multirow{2}{*}{ Mon level } & & \multirow{2}{*}{$/ \mu \mathrm{L}$} & Test food & 17 & 226.7 & \pm & 78.4 & 249.1 & \pm & 68.8 & 247.7 & \pm & 51.4 \\
\hline & & & Placebo & 17 & 201.2 & \pm & 73.3 & 217.5 & \pm & 83.0 & 213.8 & \pm & 57.9 \\
\hline \multirow{2}{*}{ Eos level } & & \multirow{2}{*}{$/ \mu \mathrm{L}$} & Test food & 17 & 100.6 & \pm & 47.2 & 98.8 & \pm & 53.7 & 111.1 & \pm & 85.8 \\
\hline & & & Placebo & 17 & 102.9 & \pm & 56.2 & 114.2 & \pm & 68.0 & 96.8 & \pm & 69.3 \\
\hline \multirow{2}{*}{ Bas level } & & \multirow{2}{*}{$/ \mu \mathrm{L}$} & Test food & 17 & 31.0 & \pm & 12.5 & 29.6 & \pm & 15.7 & 32.1 & \pm & 15.7 \\
\hline & & & Placebo & 17 & 27.2 & \pm & 24.0 & 31.0 & \pm & 17.9 & 30.0 & \pm & 17.4 \\
\hline \multirow{2}{*}{ AST } & \multirow{2}{*}{$10-40$} & \multirow{2}{*}{$\mathrm{U} / \mathrm{L}$} & Test food & 17 & 20.6 & \pm & 4.0 & 20.6 & \pm & 4.9 & 21.3 & \pm & 4.0 \\
\hline & & & Placebo & 17 & 21.1 & \pm & 4.5 & 21.5 & \pm & 4.3 & 21.4 & \pm & 4.3 \\
\hline \multirow{2}{*}{ ALT } & \multirow{2}{*}{$5-45$} & \multirow{2}{*}{$\mathrm{U} / \mathrm{L}$} & Test food & 17 & 17.2 & \pm & 5.6 & 17.8 & \pm & 8.1 & 18.4 & \pm & 6.5 \\
\hline & & & Placebo & 17 & 16.9 & \pm & 6.9 & 17.8 & \pm & 8.8 & 17.1 & \pm & 8.1 \\
\hline \multirow{2}{*}{$\gamma-\mathrm{GT}$} & \multirow{2}{*}{$<30$} & \multirow{2}{*}{$\mathrm{U} / \mathrm{L}$} & Test food & 17 & 23.1 & \pm & 12.5 & 26.1 & \pm & 18.4 & 25.7 & \pm & 16.1 \\
\hline & & & Placebo & 17 & 22.0 & \pm & 8.7 & 33.4 & \pm & 29.2 & 27.6 & \pm & 21.8 \\
\hline \multirow{2}{*}{ ALP } & & $\mathrm{U} / \mathrm{L}$ & Test food & 17 & 208.7 & \pm & 37.9 & 206.4 & \pm & 49.6 & 213.4 & \pm & 48.9 \\
\hline & $100-325$ & $\mathrm{U} / \mathrm{L}$ & Placebo & 17 & 191.3 & \pm & 42.9 & 194.3 & \pm & 63.4 & 194.4 & \pm & 59.6 \\
\hline ID & 120_240 & $\mathrm{U} / \mathrm{I}$ & Test food & 17 & 188.9 & \pm & 31.6 & 191.8 & \pm & 34.1 & 191.4 & \pm & 32.8 \\
\hline LD & $120-240$ & U/L & Placebo & 17 & 195.1 & \pm & 21.9 & 205.8 & \pm & 26.5 & 192.2 & \pm & 20.6 \\
\hline
\end{tabular}




\begin{tabular}{|c|c|c|c|c|c|c|c|c|c|c|c|c|c|}
\hline \multirow{3}{*}{ LAP } & \multicolumn{2}{|c|}{ Reference value } & \multirow{2}{*}{$\begin{array}{l}\text { Group } \\
\text { Test food }\end{array}$} & \multirow{2}{*}{$\begin{array}{l}n \\
17\end{array}$} & \multicolumn{3}{|c|}{ Pre-ingestion } & \multicolumn{3}{|c|}{ Post-12W } & \multicolumn{3}{|c|}{ Post-24W } \\
\hline & 2761 & $\mathrm{U} / \mathrm{T}$ & & & 50.9 & \pm & 5.5 & 52.8 & \pm & 7.0 & 51.3 & \pm & 6.7 \\
\hline & $3 /-01$ & U/L & Placebo & 17 & 50.9 & \pm & 6.7 & 56.0 & \pm & 12.5 & 52.2 & \pm & 11.1 \\
\hline \multirow{2}{*}{ T-Bil } & \multirow{2}{*}{$0.2-1.2$} & \multirow{2}{*}{$\mathrm{mg} / \mathrm{dL}$} & Test food & 17 & 0.8 & \pm & 0.3 & 0.8 & \pm & 0.3 & 0.8 & \pm & 0.3 \\
\hline & & & Placebo & 17 & 0.9 & \pm & 0.3 & 0.9 & \pm & 0.3 & 0.8 & \pm & 0.4 \\
\hline \multirow{2}{*}{ D-Bil } & \multirow{2}{*}{$0.0-0.2$} & \multirow{2}{*}{$\mathrm{mg} / \mathrm{dL}$} & Test food & 17 & 0.1 & \pm & 0.0 & 0.1 & \pm & 0.0 & 0.1 & \pm & 0.1 \\
\hline & & & Placebo & 17 & 0.1 & \pm & 0.1 & 0.1 & \pm & 0.1 & 0.1 & \pm & 0.0 \\
\hline \multirow{2}{*}{ I-Bil } & \multirow{2}{*}{$0.2-1.0$} & \multirow{2}{*}{$\mathrm{mg} / \mathrm{dL}$} & Test food & 17 & 0.7 & \pm & 0.3 & 0.7 & \pm & 0.2 & 0.8 & \pm & 0.3 \\
\hline & & & Placebo & 17 & 0.8 & \pm & 0.2 & 0.8 & \pm & 0.3 & 0.8 & \pm & 0.3 \\
\hline \multirow{2}{*}{$\mathrm{ChE}$} & \multirow{2}{*}{$200-452$} & \multirow{2}{*}{$\mathrm{U} / \mathrm{L}$} & Test food & 17 & 343.1 & \pm & 69.2 & 323.0 & \pm & 73.6 & 340.8 & \pm & 72.9 \\
\hline & & & Placebo & 17 & 317.7 & \pm & 53.1 & 319.2 & \pm & 61.9 & 322.0 & \pm & 67.6 \\
\hline \multirow{2}{*}{ ZTT } & \multirow{2}{*}{$2.0-12.0$} & \multirow{2}{*}{$\mathrm{U}$} & Test food & 17 & 6.8 & \pm & 2.1 & 7.1 & \pm & 2.2 & 7.1 & \pm & 1.9 \\
\hline & & & Placebo & 17 & 5.6 & \pm & 2.5 & 6.2 & \pm & 2.7 & 6.3 & \pm & 3.1 \\
\hline \multirow{2}{*}{$\mathrm{TP}$} & \multirow{2}{*}{$6.7-8.3$} & \multirow{2}{*}{$\mathrm{g} / \mathrm{dL}$} & Test food & 17 & 7.1 & \pm & 0.3 & 7.0 & \pm & 0.3 & 6.9 & \pm & 0.4 \\
\hline & & & Placebo & 17 & 7.0 & \pm & 0.3 & 6.9 & \pm & 0.3 & 6.7 & \pm & 0.3 \\
\hline \multirow{2}{*}{ UN } & \multirow{2}{*}{$8.0-20.0$} & \multirow{2}{*}{$\mathrm{mg} / \mathrm{dL}$} & Test food & 17 & 12.4 & \pm & 3.1 & 13.6 & \pm & 2.8 & 14.5 & \pm & 3.7 \\
\hline & & & Placebo & 17 & 12.8 & \pm & 2.2 & 13.9 & \pm & 2.9 & 13.2 & \pm & 2.3 \\
\hline \multirow{2}{*}{ CRE } & & & Test food & 17 & 0.6 & \pm & 0.1 & 0.6 & \pm & 0.1 & 0.7 & \pm & 0.1 \\
\hline & $0.47-0.19$ & $\mathrm{mg} / \mathrm{dL}$ & Placebo & 17 & 0.6 & \pm & 0.1 & 0.6 & \pm & 0.1 & 0.6 & \pm & 0.1 \\
\hline Ju & 25,70 & $\mathrm{mo} / \mathrm{dI}$ & Test food & 17 & 4.6 & \pm & 0.9 & 4.4 & \pm & 0.7 & 4.6 & \pm & 0.7 \\
\hline UA & $2.5-1.0$ & mg/dL & Placebo & 17 & 4.2 & \pm & 0.8 & 4.2 & \pm & 0.7 & 4.2 & \pm & 0.8 \\
\hline$C V$ & 150 & U/T & Test food & 17 & 97.5 & \pm & 48.1 & 96.1 & \pm & 47.1 & 101.0 & \pm & 48.6 \\
\hline CK & $40-150$ & U/L & Placebo & 17 & 96.1 & \pm & 33.5 & 92.2 & \pm & 35.3 & 98.6 & \pm & 38.6 \\
\hline & & & Test food & 17 & 142.5 & \pm & 1.5 & 141.1 & \pm & 2.4 & 141.0 & \pm & 1.7 \\
\hline $\mathrm{Na}$ & $13 /-14 /$ & $\mathrm{mEq} / \mathrm{L}$ & Placebo & 17 & 142.4 & \pm & 1.7 & 140.7 & \pm & 2.4 & 141.2 & \pm & 2.0 \\
\hline & & & Test food & 17 & 4.1 & \pm & 0.3 & 3.9 & \pm & 0.2 & 4.1 & \pm & 0.3 \\
\hline $\mathrm{K}$ & $3.5-5.0$ & $\mathrm{mEq} / \mathrm{L}$ & Placebo & 17 & 4.2 & \pm & 0.5 & 3.9 & \pm & 0.4 & 4.1 & \pm & 0.3 \\
\hline
\end{tabular}




\begin{tabular}{|c|c|c|c|c|c|c|c|c|c|c|}
\hline \multirow{3}{*}{$\mathrm{Cl}$} & \multicolumn{2}{|c|}{ Reference value } & \multirow{2}{*}{$\begin{array}{l}\text { Group } \\
\text { Test food }\end{array}$} & \multirow{2}{*}{$\begin{array}{l}n \\
17\end{array}$} & \multicolumn{2}{|c|}{ Pre-ingestion } & \multicolumn{2}{|c|}{ Post-12W } & \multicolumn{2}{|c|}{ Post-24W } \\
\hline & & & & & 101.8 & \pm 1.4 & 100.9 & \pm 2.9 & 101.4 & \pm 2.5 \\
\hline & $98-108$ & $\mathrm{mEq} / \mathrm{L}$ & Placebo & 17 & 102.5 & \pm 1.4 & 101.6 & \pm 2.3 & 102.2 & \pm 1.8 \\
\hline \multirow{2}{*}{$\mathrm{Ca}$} & \multirow{2}{*}{ 8.4-10.4 } & \multirow{2}{*}{$\mathrm{mg} / \mathrm{dL}$} & Test food & 17 & 9.2 & \pm 0.2 & 8.9 & \pm 0.3 & 9.1 & \pm 0.3 \\
\hline & & & Placebo & 17 & 9.1 & \pm 0.3 & 9.1 & \pm 0.3 & 9.0 & \pm 0.3 \\
\hline \multirow{2}{*}{ IP } & \multirow{2}{*}{$2.5-4.5$} & \multirow{2}{*}{$\mathrm{mg} / \mathrm{dL}$} & Test food & 17 & 3.7 & \pm 0.6 & 4.0 & \pm 0.5 & 3.5 & \pm 0.4 \\
\hline & & & Placebo & 17 & 3.7 & \pm 0.4 & 3.9 & \pm 0.5 & 3.3 & \pm 0.5 \\
\hline \multirow{2}{*}{$\mathrm{Fe}$} & \multirow{2}{*}{$40-180$} & \multirow{2}{*}{$\mu \mathrm{g} / \mathrm{dL}$} & Test food & 17 & 104.2 & \pm 19.9 & 103.6 & \pm 32.0 & 113.5 & \pm 31.8 \\
\hline & & & Placebo & 17 & 95.2 & \pm 29.7 & 106.8 & \pm 32.6 & 102.5 & \pm 35.5 \\
\hline \multirow{2}{*}{ AMY } & \multirow{2}{*}{$40-122$} & \multirow{2}{*}{$\mathrm{U} / \mathrm{L}$} & Test food & 17 & 80.1 & \pm 35.8 & 77.2 & \pm 32.7 & 84.5 & \pm 40.5 \\
\hline & & & Placebo & 17 & 77.6 & \pm 26.2 & 79.0 & $\pm \quad 30.9$ & 80.8 & $\pm \quad 37.7$ \\
\hline \multirow{2}{*}{$\mathrm{TC}$} & \multirow{2}{*}{$120-219$} & \multirow{2}{*}{$\mathrm{mg} / \mathrm{dL}$} & Test food & 17 & 229.6 & \pm 36.8 & 222.3 & \pm 43.1 & 235.3 & \pm 42.2 \\
\hline & & & Placebo & 17 & 242.9 & \pm 30.3 & 242.8 & $\pm \quad 31.5$ & 248.7 & $\pm \quad 31.1$ \\
\hline \multirow{2}{*}{ HDL-C } & \multirow{2}{*}{$40-95$} & \multirow{2}{*}{$\mathrm{mg} / \mathrm{dL}$} & Test food & 17 & 75.6 & \pm 18.1 & 72.7 & \pm 18.4 & 75.8 & \pm 20.3 \\
\hline & & & Placebo & 17 & 83.6 & \pm 20.2 & 83.2 & \pm 21.3 & 87.6 & \pm 21.8 \\
\hline \multirow{2}{*}{ LDL-C } & \multirow{2}{*}{$65-139$} & \multirow{2}{*}{$\mathrm{mg} / \mathrm{dL}$} & Test food & 17 & 134.2 & $\pm \quad 30.7$ & 130.5 & $\pm \quad 36.0$ & 134.5 & \pm 34.2 \\
\hline & & & Placebo & 17 & 143.2 & \pm 29.0 & 144.0 & \pm 31.6 & 141.9 & $\pm \quad 30.5$ \\
\hline \multirow{2}{*}{ TG } & \multirow{2}{*}{ 30-149 } & \multirow{2}{*}{$\mathrm{mg} / \mathrm{dL}$} & Test food & 17 & 95.3 & \pm 56.6 & 102.6 & \pm 55.8 & 96.2 & \pm 55.9 \\
\hline & & & Placebo & 17 & 77.4 & \pm 34.2 & 79.4 & \pm 37.5 & 77.1 & $\pm \quad 39.1$ \\
\hline Glu & $70-100$ & $\mathrm{mo} / \mathrm{dI}$ & Test food & 17 & 83.9 & \pm 10.8 & 85.9 & \pm 6.5 & 83.4 & \pm 8.4 \\
\hline Giu & $10-109$ & mg/aL & Placebo & 17 & 86.8 & \pm 9.6 & 85.4 & \pm 8.5 & 86.4 & \pm 10.4 \\
\hline $\mathrm{Hb} \Delta 1 \mathrm{c}$ & 4662 & or & Test food & 17 & 5.6 & \pm 0.2 & 5.6 & \pm 0.2 & 5.5 & \pm 0.2 \\
\hline HbAIc & $4.0-6.2$ & $\%$ & Placebo & 17 & 5.5 & \pm 0.2 & 5.4 & $\pm \quad 0.2$ & 5.4 & \pm 0.3 \\
\hline GA & $123-165$ & $o$ & Test food & 17 & 14.4 & \pm 1.7 & 15.0 & \pm 1.7 & 14.7 & \pm 1.5 \\
\hline GA & $12.3-10.5$ & $\%$ & Placebo & 17 & 14.2 & \pm 1.3 & 14.8 & $\pm \quad 1.3$ & 14.5 & $\pm \quad 1.4$ \\
\hline
\end{tabular}

Values are mean \pm SD

${ }^{\#} P<0.05$ (vs. Placebo group) 
Table 6. Changes in urinalysis parameters (safety assessment)

\begin{tabular}{|c|c|c|c|c|c|c|}
\hline & $\begin{array}{l}\text { Reference } \\
\text { value }\end{array}$ & Group & $N$ & Pre-ingestion & Post-12W & Post-24W \\
\hline \multirow{2}{*}{ Protein } & \multirow{2}{*}{$(-)$} & Test food & 17 & $(-): n=17$ & $(-): n=16,( \pm): n=1$ & $(-): n=15,( \pm): n=2$ \\
\hline & & Placebo & 17 & $(-): n=17$ & $(-): n=17$ & $(-): n=17$ \\
\hline \multirow{2}{*}{ Glucose } & \multirow{2}{*}{$(-)$} & Test food & 17 & $(-): n=17$ & $(-): n=17$ & $(-): n=17$ \\
\hline & & Placebo & 17 & $(-): n=17$ & $(-): n=17$ & $(-): n=17$ \\
\hline \multirow{2}{*}{ Urobilinogen } & \multirow{2}{*}{$( \pm)$} & Test food & 17 & $( \pm): n=17$ & $( \pm): n=17$ & $( \pm): n=17$ \\
\hline & & Placebo & 17 & $( \pm): n=17$ & $( \pm): n=17$ & $( \pm): n=17$ \\
\hline \multirow{2}{*}{ Bilirubin } & \multirow{2}{*}{$(-)$} & Test food & 17 & $(-): n=17$ & $(-): n=17$ & $(-): n=17$ \\
\hline & & Placebo & 17 & $(-): n=17$ & $(-): n=17$ & $(-): n=17$ \\
\hline \multirow{2}{*}{ Ketone } & \multirow{2}{*}{$(-)$} & Test food & 17 & $(-): n=16,(+): n=1$ & $(-): n=17$ & $(-): n=17$ \\
\hline & & Placebo & 17 & $(-): n=17$ & $(-): n=17$ & $(-): n=17$ \\
\hline \multirow{2}{*}{$\mathrm{pH}$} & \multirow{2}{*}{$5.0-7.5$} & Test food & 17 & $(5.0-7.5): n=16,(8.0): n=1$ & $(5.0-7.5): n=17$ & $(5.0-7.5): n=17$ \\
\hline & & Placebo & 17 & $(5.0-7.5): n=17$ & $(5.0-7.5): n=17$ & $(5.0-7.5): n=17$ \\
\hline \multirow{2}{*}{ Occult blood } & \multirow{2}{*}{$(-)$} & Test food & 17 & $(-): n=17$ & $(-): n=17$ & $(-): n=16,( \pm): n=1$ \\
\hline & & Placebo & 17 & $(-): n=17$ & $(-): n=16,( \pm): n=1$ & $(-): n=15,( \pm): n=2$ \\
\hline
\end{tabular}

The number of subjects with each result is shown. 


\section{DISCUSSION}

Osteoporosis is characterized by disrupted homeostasis of bone metabolism and leads a prolonged state of bone resorption. Women show a higher prevalence of osteoporosis than men, and it has been estimated that $70 \%$ of osteoporosis patients over the age of 40 are female [2]. Postmenopausal women exhibit accelerated osteoclast differentiation associated with estrogen deficiency, which leads to decreased bone density by increasing the rate of bone turnover [20]. In addition, the intestinal absorption rate of calcium decreases rapidly from immediately after menopause [21]. Therefore, it is important to take precautions against osteoporosis both before and after menopause in women $[22,23]$.

The present study aimed to investigate whether the ingestion of maltobionic acid elicited improvement in BMD in women aged 50-69 years who were at least 1 year past the onset of natural menopause. BMC of the total anterior surface of the lumbar vertebrae continued to decline by $-0.30 \mathrm{~g}$ at post- $12 \mathrm{~W}$ and $-0.88 \mathrm{~g}$ at post- $24 \mathrm{~W}$ of ingestion in the Placebo group compared with that observed pre-ingestion. In contrast, in the Test food group, the corresponding values increased by $+0.31 \mathrm{~g}$ at post- $12 \mathrm{~W}$ and $+0.85 \mathrm{~g}$ at post- $24 \mathrm{~W}$ of ingestion, compared with those observed preingestion in the Placebo group. Intake of maltobionic acid contained in the test food increased calcium absorption and the amount of calcium in the femur when tested in a rat model [13]. Therefore, it is presumed that the intestinal absorption of calcium was enhanced by the ingestion of maltobionic acid, and that the efficient absorption of calcium via the intestine, without being excreted, contributed to the maintenance and increase in BMC.

Among the bone metabolism markers analyzed in this study, TRACP-5b, DPD, s-NTx, and uNTx are bone resorption markers, and OC and BAP are bone formation markers. In particular, $\mathrm{DPD}$, which is a bone resorption marker, is widely used as a non-invasive marker in medical practice to diagnose osteoporosis and confirm therapeutic effects $[24,25]$. In the current study, subjects in the Test food group showed consistently lower DPD values from pre-ingestion to post$24 \mathrm{~W}$ than subjects in the Placebo food group. Maltobionic acid contained in the test food has been confirmed to inhibit the differentiation of osteoclasts in an in vitro study (unpublished data). This suggests that maltobionic acid suppressed osteoclast differentiation and bone resorption, thereby attenuating the release of DPD. The findings indicate that not only was the absorption of calcium enhanced by the intake of maltobionic acid, but homeostasis of bone metabolism was also improved by suppressing bone resorption, which contributed to the maintenance of bone density. Of note, the primary outcome of this study, i.e. BMD at the front of the lumbar spine, is widely used as a diagnostic indicator of osteoporosis and fracture risk [15]. BMD of the total anterior surface of the lumbar vertebrae was significantly higher in the Test food group than in the Placebo group from pre-ingestion to post-24W. Soy isoflavones are widely used as therapeutic agents for osteoporosis because they have hormone-like effects and improve bone metabolism [26, 27]. An interventional study examining the effects of intake of isoflavones in postmenopausal women identified an increased BMD of $0.015 \mathrm{~g} / \mathrm{cm}^{2} 24$ weeks after ingestion, suggesting that they exhibit efficacy for the treatment of osteoporosis in this population [29]. Thus, the amount of change 0.015 $\mathrm{g} / \mathrm{cm}^{2}$ in bone mineral density is clinically meaningful. BMD of the total anterior surface of the lumbar vertebrae in this study decreased consistently in the Placebo group up to post-24W, with a reduction of $0.013 \mathrm{~g} / \mathrm{cm}^{2}$. In contrast, an increase in BMD of $+0.002 \mathrm{~g} / \mathrm{cm}^{2}$ was observed in the 
test food group post- $24 \mathrm{~W}$ compared with that before ingestion. Therefore, it is believed that the ingestion of the test food inhibited BMD loss (around $0.015 \mathrm{~g} / \mathrm{cm}^{2}$, which is considered clinically meaningful) that may occur in postmenopausal women. The same results were confirmed in the intervention test with calcium salts mainly composed of maltobionic acid [14], indicating that maltobionic acid contributes to maintenance and improvement of bone density, regardless of whether they are ingested as mineral salts or in a mineral-free state. However, further studies are necessary to elucidate the mechanism by which the intake of maltobionic acid improves bone density. This study has several limitations. First, despite the results suggesting that maltobionic acid inhibits bone resorption, no differences were seen in the bone resorption markers TPACP-5b or u-NYx and s-NTx. A detailed assessment of the effect on bone resorption requires a review of the duration of the intervention and timing of the measurements. Second, because the BDHQ used in the dietary survey is a questionnaire that collects information on the dietary habit followed in the previous month, the effect of the intake of calcium on BMD may not have been accurately reflected. Although calcium intake before and during the intervention period did not differ significantly between the groups, future studies should aim to examine the effect of the test food on improving bone density in more detail by including dietary surveys that record the subjects' daily meals.

The safety of the test food utilized in this study was assessed through physical measurements, physiological testing, urinalysis, and peripheral blood tests conducted at the pre-ingestion, post$12 \mathrm{~W}$, and post- $24 \mathrm{~W}$ time points. Some of the urinalysis results were false positives, and there were no significant inter-group differences; therefore, we concluded that the changes were of no medical significance. Although some items among the physical measurements, physiological tests, and peripheral blood tests showed significant intra- and inter-group differences, all values were within the standard range or only slight changes were observed. Based on these findings, we concluded that these changes were of no medical significance. Based on the safety assessment results, we found no clinically meaningful changes or adverse events that were thought to have been caused by ingestion of the test food over the 24 -week period. Thus, we concluded that the test food did not pose any safety-related concerns in the study population.

\section{CONCLUSION}

Ingestion of the test food - corn syrup containing maltobionic acid - over a 24-week period led to the maintenance and increase of BMD, BMC, and YAM values in Japanese women aged 50-69 years who were at least 1 year past the onset of natural menopause. The results indicate that maltobionic acid can contribute to the prevention of osteoporosis by maintaining and increasing bone density in this age group. The results of this study additionally demonstrate the safety of its long-term ingestion over a 24-week period. Therefore, continuous intake of maltobionic acid in healthy Japanese adult women was found to improve bone mineral density and may contribute to the prevention of osteoporosis.

List of Abbreviations: BAP, bone-specific alkaline phosphatase; BDHQ, brief-type selfadministered diet history questionnaire; BMC, bone mineral content; BMD, bone mineral density; DEXA, dual-energy X-ray absorptiometry method; DPD, deoxypyridinoline; NTx, type I collagen 
cross-linked N-telopeptides; OC, osteocalcin; SD, standard deviation; TRACP-5b, tartrateresistant acid phosphatase 5b; YAM, young adult mean

Competing Interests: Daiki Suehiro and Ken Fukami are employees of San-ei Sucrochemical Co., Ltd. San-ei Sucrochemical Co., Ltd. is the supplier of a food product containing maltobionic acid.

Author Contributions: Daiki Suehiro, Ken Fukami, and Tsuyoshi Takara designed the research protocol. Daiki Suehiro and Ken Fukami provided test and placebo foods. Tsuyoshi Takara is was the principal investigator of for this study and analyzed the physical data for evaluation of adverse events. Daiki Suehiro wrote the manuscript. Ken Fukami and Tsuyoshi Takara reviewed and edited the manuscript. Daiki Suehiro had primary responsibility for the final content. All authors read and approved the final version of the manuscript.

Acknowledgements and Funding: The authors thank the staff of Takara Clinic and ORTHOMEDICO Inc. who provided valuable assistance with the study and clinical testing.

\section{REFERENCES:}

1. NIH Consensus Development Panel on Osteroporosis, Diagnosis, and Therapy: Osteoporosis prevention, diagnosis, and therapy. JAMA 2001, 285(6):785-795.

2. Yoshimura N, Muraki S, Oka H, Mabuchi A, En-Yo Y, Yoshida M, Saika A, et al.: Prevalence of knee osteoarthritis, lumbar spondylosis, and osteoporosis in Japanese men and women: the research on osteoarthritis/osteoporosis against disability study. J Bone Miner Metab 2009, 27(5):620-628.

3. Yoshimura N, Muraki S, Oka H, Kawaguchi H, Nakamura K, Akune T: Cohort profile: research on Osteoarthritis/Osteoporosis Against Disability study. Int $\mathrm{J}$ Epidemiol 2010, 39(4):988-995.

4. Silverman SL, Minshall ME, Shen W, Harper KD, Xie S: The relationship of healthrelated quality of life to prevalent and incident vertebral fractures in postmenopausal women with osteoporosis: results from the Multiple Outcomes of Raloxifene Evaluation Study. Arthritis Rheum 2001, 44(11):2611-2619.

5. Bliuc D, Nguyen ND, Milch VE, Nguyen TV, Eisman JA, Center JR: Mortality risk associated with low-trauma osteoporotic fracture and subsequent fracture in men and women. JAMA 2009, 301(5):513-521.

6. Fujiwara S, Kasagi F, Masunari N, Naito K, Suzuki G, Fukunaga M: Fracture prediction from bone mineral density in Japanese men and women. J Bone Miner Res 2003, 18(8):1547-1553.

7. Cumming RG, Nevitt MC: Calcium for prevention of osteoporotic fractures in postmenopausal women. J Bone Miner Res 1997, 12(9):1321-1329.

8. Welten DC, Kemper HC, Post GB, van Staveren WA: A meta-analysis of the effect of calcium intake on bone mass in young and middle-aged females and males. J Nutr 1995, 125(11):2802-2813. 
9. Yaegashi Y, Onoda T, Tanno K, Kuribayashi T, Sakata K, Orimo H: Association of hip fracture incidence and intake of calcium, magnesium, vitamin D, and vitamin K. Eur J Epidemiol 2008, 23(3):219-225.

10. Spencer H, Scheck J, Lewin I, Samachson J: Comparative absorption of calcium from calcium gluconate and calcium lactate in man. J Nutr 1966, 89(3):283-292.

11. Hamalainen MM: Bone repair in calcium-deficient rats: comparison of xylitol + calcium carbonate with calcium carbonate, calcium lactate and calcium citrate on the repletion of calcium. J Nutr 1994, 124(6):874-881.

12. Suehiro D, Okada M, Fukami K, Otsuka M, Nakagawa T, Hayakawa T: Effect of Calcium Maltobionate on Enhancement of Calcium Absorption in Rats. Luminacoids Res 2017, 21(1):1-7.

13. Suehiro D, Okada M, Fukami K, Nakagawa T, Hayakawa T: Maltobionic acid enhances intestinal absorption of calcium and magnesium in rats. Biosci Biotechnol Biochem 2019, 83(9):1766-1773.

14. Fukami K, Suehiro D, Takara T: Safety evaluation of long-term intake and effects on bone mineral density of corn syrup solids containing maltobionic acid (maltobionic acid calcium salt) in healthy Japanese women-a randomized double-blind placebocontrolled study. Japanese Pharmacology and Therapeutics 2019, 47(2):229-245.

15. Soen S, Fukunaga M, Sugimoto T, Sone T, Fujiwara S, Endo N, Gorai I, et al.: Diagnostic criteria for primary osteoporosis: year 2012 revision. J Bone Miner Metab 2013, 31(3):247-257.

16. Kobayashi S, Murakami K, Sasaki S, Okubo H, Hirota N, Notsu A, Fukui M, et al.: Comparison of relative validity of food group intakes estimated by comprehensive and brief-type self-administered diet history questionnaires against $16 \mathrm{~d}$ dietary records in Japanese adults. Public Health Nutr 2011, 14(7):1200-1211.

17. Kobayashi S, Honda S, Murakami K, Sasaki S, Okubo H, Hirota N, Notsu A, et al.: Both comprehensive and brief self-administered diet history questionnaires satisfactorily rank nutrient intakes in Japanese adults. J Epidemiol 2012, 22(2):151159.

18. Scariano JK, Glew RH, Bou-Serhal CE, Clemens JD, Garry PJ, Baumgartner RN: Serum levels of cross-linked $\mathrm{N}$-telopeptides and aminoterminal propeptides of type I collagen indicate low bone mineral density in elderly women. Bone 1998, 23(5):471-477.

19. Shiraki M, Ito H, Hatano S, Shichida K, Sugiura M, Suzuki Y, Orimo H, et al.: Effect of Aging on Bone Mineral Content. Part III. The difference in the loss of bone mineral with aging between male and female. Nippon Ronen Igakkai Zasshi 1984, 21(1):8-13.

20. Riggs BL, Khosla S, Melton LJ 3rd: Sex steroids and the construction and conservation of the adult skeleton. Endocr Rev 2002, 23(3):279-302.

21. Nordin BE, Need AG, Morris HA, O'Loughlin PD, Horowitz M: Effect of age on 
calcium absorption in postmenopausal women. Am J Clin Nutr 2004, 80(4):9981002.

22. Hien VT, Khan NC, Mai le B, Lam NT, Phuong TM, Nhung BT, Nhien NV, et al.: Effect of community-based nutrition education intervention on calcium intake and bone mass in postmenopausal Vietnamese women. Public Health Nutr 2009, 12(5):674-679.

23. Martyn-St James M, Carroll S: Meta-analysis of walking for preservation of bone mineral density in postmenopausal women. Bone 2008, 43(3):521-531.

24. Robins SP, Black D, Paterson CR, Reid DM, Duncan A, Seibel MJ: Evaluation of urinary hydroxypyridinium crosslink measurements as resorption markers in metabolic bone diseases. Eur J Clin Invest 1991, 21(3):310-315.

25. Uebelhart D, Schlemmer A, Johansen JS, Gineyts E, Christiansen C, Delmas PD: Effect of menopause and hormone replacement therapy on the urinary excretion of pyridinium cross-links. J Clin Endocrinol Metab 1991, 72(2):367-373.

26. Setchell KD, Cassidy A: Dietary isoflavones: biological effects and relevance to human health. J Nutr 1999, 129(3):758S-767S.

27. Taku K, Melby MK, Kurzer MS, Mizuno S, Watanabe S, Ishimi Y: Effects of soy isoflavone supplements on bone turnover markers in menopausal women: systematic review and meta-analysis of randomized controlled trials. Bone 2010, 47(2):413-423.

28. Kovacs AB: Efficacy of ipriflavone in the prevention and treatment of postmenopausal osteoporosis. Agents Actions 1994, 41(1-2):86-87. 